\title{
INVESTIGATION ON THERMAL DEFORMATION IN LASER ADDITIVE MANUFACTURING
}

\author{
Y. Kuroiwa1 ${ }^{1}$ D. Kono ${ }^{1 *}$, Y. Oda ${ }^{2}$ \\ ${ }^{1}$ Kyoto university, C3, Kyotodaigaku Katsura, Kyoto, 815-8540, Japan \\ 2 DMG MORI CO., LTD., 2-35-16 Meieki, Nakamura-ku, Nagoya, Aichi, 450-0002, Japan \\ *Corresponding author; e-mail: kono@prec.kyoto-u.ac.jp
}

\begin{abstract}
In metal additive manufacturing, a metal material is melted by a concentrated heat source such as a laser. Therefore, thermal deformation occurs in the fabrication, which causes deterioration of shape accuracy and crack of the workpiece. In this study, a method to systematically reduce the thermal deformation was discussed. The mechanism of thermal deformation caused by stacking and lining up the bead was investigated using finite element simulations and experiments. Based on the obtained results and thermal deformation theory in welding, a method to reduce the thermal deformation was proposed and the validity of the method was demonstrated by simulation.
\end{abstract}

\section{Keywords:}

Additive manufacturing; Thermal deformation; Simulation; Directed; Energy Deposition

\section{INTRODUCTION}

Metal additive manufacturing is a method of manufacturing products by fabricating metals based on 3D slice data of the target shape. Metal additive manufacturing is able to manufacture products with complicated shapes that cannot be manufactured by removal processing such as cutting. This advantage improves the performance of products, reduces costs, and shortens the development period. Therefore, its utilization is attracting attention in fields such as the aircraft industry and the medical industry where highmix low-volume production is common [Klocke 2014] [Bartolo 2012] [Thompson 2016]. In recent years, additive manufacturing machines that can handle large-sized workpieces have been developed, and are expected to be applied to a wide range of industries.

In metal additive manufacturing, a metal material is melted and solidified by a centralized heat source such as laser [ $\mathrm{L}$ 2018]. As a result, thermal strain may occur in the workpiece which leads to deterioration of shape accuracy, residual stress and workpiece cracks [Zhang 2018] Therefore, in metal additive manufacturing, it is necessary to select appropriate fabrication conditions that can suppress thermal strain. However, at present, based on the experience of the operator, thermal strain and cracking are avoided by changing the orientation of the workpiece and the fabricating route by trial and error. A method for systematically determining fabricating conditions that can avoid thermal strain and cracking is demanded.

So far, many studies have been conducted with the aim of reducing the thermal strain of the workpiece. Typical means include selection of a fabrication path pattern [Cheng 2016], adjustment of fabrication parameters such as laser power and laser scanning speed [Simson 2017], and heat treatment after stacking [Dong 2014]. However, these studies are not systematic but trial and error.

Regarding the generated thermal strain and residual stress, the theory of laser welding can be applied because the metal additive manufacturing is similar to laser welding. In the welding field, empirical formulas have been established for deformation and stress states due to welding heat [Verhaeghe 1999]. However, these formulas are for one welding line, and cannot handle additive manufacturing because multiple beads are placed horizontally and stacked vertically in additive manufacturing.

The final goal of this study is to propose a systematic method for reducing out-of-plane thermal workpiece deformation. Experiments and simulations are conducted to investigate the in-plane and out-of-plane thermal displacement of the workpiece which are the thermal deformation particularly in additive manufacturing. Then a conceptual method based on the investigation is proposed. The validity of the proposed method is evaluated by simulation.

\section{EFFECTS OF IN PLANE FABRICATION ON THERMAL DEFORMATION}

As shown in Fig1, additive manufacturing consists of in plane and out of plane fabrication. Thus, the workpiece thermal deformation in these two fabrications are focussed in this study. In this section, experiment and simulation 
were performed to investigate the effect of in plane fabrication on the thermal deformation.

\subsection{Machine tools used in the experiment}

Directed Energy Deposition (DED) was used in the experiment in this study. Fig. 2 shows the schematic diagram of DED. In the machine, the laser and metal powder are supplied through the coaxial nozzle, and powder is supplied to the surface to be fabricated from the circular powder supply nozzle that surrounds the laser irradiation port. Tab. 1 shows the laser conditions in the experiment. In this study, JIS SUS316L stainless steel was used as the metal powder. The metal powder grain size was 50-100 $\mu \mathrm{m}$.

\subsection{Experiment method}

Fig. 3 shows the geometry of substrate and fabricated workpiece. Three in plane beads were fabricated on the upper surface of the substrate, and the displacement of the upper surface of the substrate in the $\mathbf{Z}$ direction was obtained by experiments and simulation

The substrate was fixed to a machine table with a M5 cap screw. The material of the substrate was JIS SUS316L stainless steel. In order to confirm the validity of the thermal conditions in the simulation, the substrate temperature was measured using a K-type thermocouple. The thermocouples were fixed on the plate by spot welding.

Three beads were fabricated in the plane in order toward the positive $X$ direction. The laser scanning by DED proceeded in the positive $Y$ direction. The profile of the substrate surface was measured when the first bead and third bead was fabricated using a laser displacement sensor. The thermal displacement of the substrate was obtained by subtracting the initial substrate profile before fabrication from the measured profile. The specifications of the laser displacement sensor are shown in Tab. 2.

Lines $A A^{\prime}$ and BB' shown in Fig. 3 are the measurement position of the substrate profile. $A^{\prime}$ and $B$ are the same point. The profile near the side of the substrate is measured because the deformation is larger.

\subsection{Simulation method}

Simulation was performed using the finite element method (FEM). Transient thermal analysis and static structure analysis were combined. Three rectangular blocks were generated one by one as beads in-plane on the substrate. The hatched area with $7 \mathrm{~mm}$ diameter around the M5 screw in Fig. 3 was fixed. The thermal displacement of the substrate was obtained at the first and third bead fabrication.

The laser heat was applied just after each bead was generated. In order to save computational resources, the lump heat in one bead scan was applied to the top surface of each bead. Thus, the movement of the easer spot was not considered in this simulation.

The laser heat and its duration time were determined to represent the experimental condition. The duration time of the laser heat was set to $0.18 \mathrm{~s}$ that was obtained by dividing $3 \mathrm{~mm}$ laser spot diameter by $1000 \mathrm{~mm} / \mathrm{min}$ scanning speed. In contrast, the deposition time for one bead was $1.8 \mathrm{~s}$ in the experiment. However, because the movement of the laser spot is not considered, the $1.8 \mathrm{~s}$ laser duration time is too long for the simulation to represent the quick temperature variation in deposition. The deposition process can be more properly represented by superposition of deposition at one laser spot.

To obtain the laser heat, the heat balance at one local spot in the bead is considered. The heat transfers to the part with lower temperature in the experiment because the

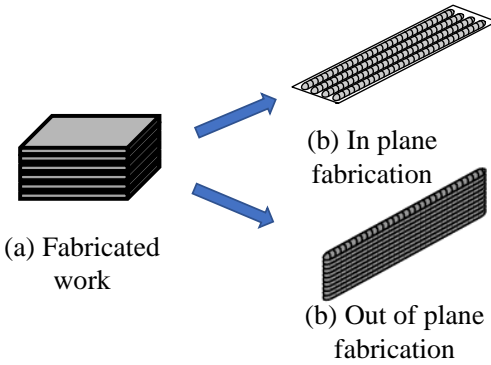

Fig. 1: Two types of fabrication.

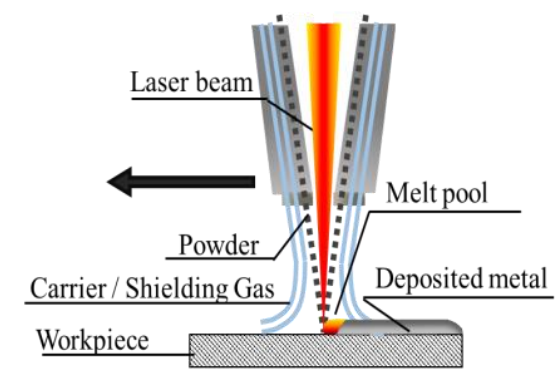

Fig. 2: Schematic of Directed Energy Deposition

Tab. 1: Laser beam conditions in experiment.

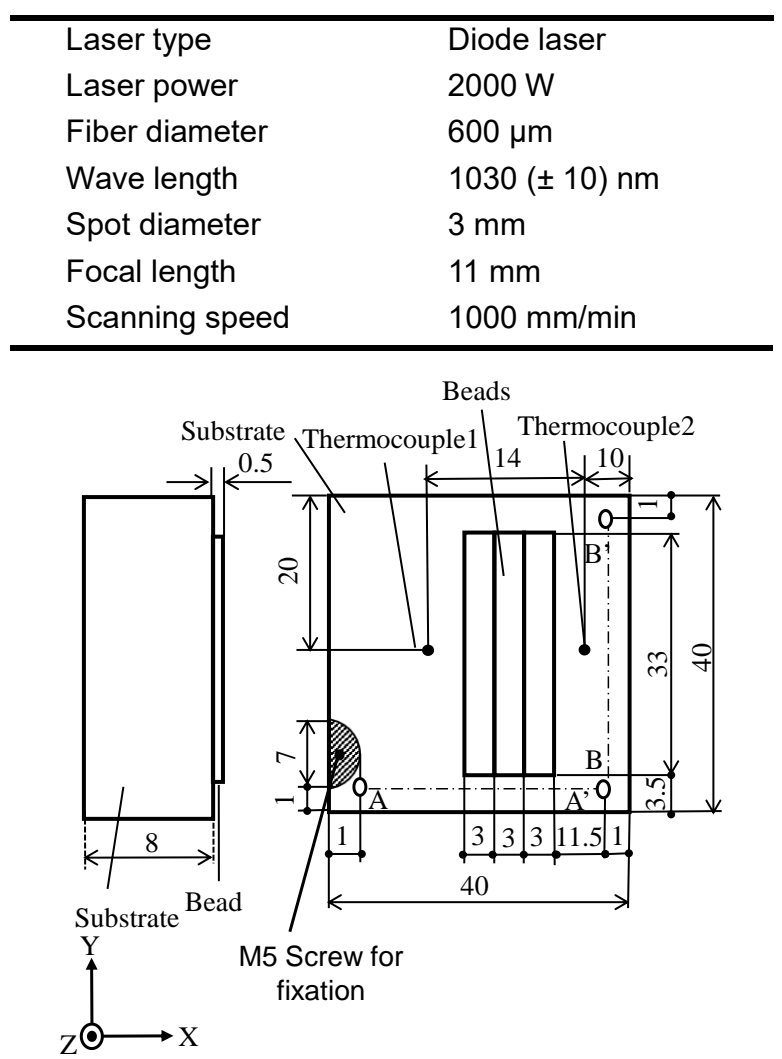

Fig. 3: Geometry of substrate and fabrication in plane.

Tab. 2 : Specifications of laser displacement sensor

\begin{tabular}{ll} 
Working distance & $60 \mathrm{~mm}$ \\
Measurement range & $60 \pm 8 \mathrm{~mm}$ (height) \\
& $15 \mathrm{~mm}$ (width) \\
Light source wavelength & $405 \mathrm{~nm}$ \\
Spot diameter & $20 \mu \mathrm{m}$ \\
Repeatability & $5 \mu \mathrm{m}$ (width) \\
& $0.4 \mu \mathrm{m}$ (height) \\
\hline
\end{tabular}


Tab. 3 thermal condition considered in simulation.

\begin{tabular}{cc}
\hline Heat convection & $8 \mathrm{~W} / \mathrm{m}^{2}$ \\
Emissivity & 0.1 \\
Heat flow considering & $10000 \mathrm{~W}$ \\
absorption rate & $0.18 \mathrm{~s}$ \\
Heating time $/$ bead &
\end{tabular}

Tab. 4 Material parameters of SUS316L depending on temperature.

\begin{tabular}{ccll}
\hline $\begin{array}{c}\text { Temperature } \\
\mathrm{K}\end{array}$ & $\begin{array}{c}\text { Coefficient } \\
\text { of } \\
\text { Thermal } \\
\text { Expantion } \\
/ \mathrm{K}\end{array}$ & $\begin{array}{c}\text { Young's } \\
\text { Modulus } \\
\mathrm{Pa}\end{array}$ & $\begin{array}{c}\text { Yield } \\
\text { Strength } \\
\mathrm{Pa}\end{array}$ \\
& 1.47 & 1.95 & 2.35 \\
298 & $\times 10^{-5}$ & $\times 10^{11}$ & $\times 10^{8}$ \\
& 1.69 & 1.80 & 1.83 \\
573 & $\times 10^{-5}$ & $\times 10^{11}$ & $\times 10^{8}$ \\
& 1.83 & 1.55 & 1.26 \\
873 & $\times 10^{-5}$ & $\times 10^{11}$ & $\times 10^{8}$ \\
& 1.92 & 1.17 & 6.93 \\
1173 & $\times 10^{-5}$ & $\times 10^{11}$ & $\times 10^{7}$ \\
& 1.99 & 5.10 & 1.24 \\
1473 & $\times 10^{-5}$ & $\times 10^{10}$ & $\times 10^{7}$ \\
\hline
\end{tabular}

temperature of the workpiece ahead and far behind from the laser spot is lower than that of laser spot. However, in contrast, such heat transfer does not occur in this simulation because the entire bead is heated at the same time. Thus, the laser heat input was decreased from the experimental heat input in this simulation by multiplying the area ratio between the laser spot and $3 \mathrm{~mm} \times 3 \mathrm{~mm}$ square that the laser spot passed through locally. The input heat to a bead $H_{\text {total }}$ in the simulation was obtained by the following equation.

$H_{\text {total }}=2000 \mathrm{~W} \times \frac{1.5 \mathrm{~mm} \times 1.5 \mathrm{~mm} \times \pi}{3 \mathrm{~mm} \times 3 \mathrm{~mm}} \times 10$

The laser heat was calculated to $15555 \mathrm{~W}$ from Eq. (1). According to J.P.Kruth et al's work, the absorption rate of laser heat for SUS316L powder was assumed to be 0.64 [Kruth 2003]. Therefore the heat input amount of each bead was $10000 \mathrm{~W}$, which was $15555 \mathrm{~W}$ multiplied by the absorption rate 0.64 .

The simulation was conducted by a commercial FEM software ANSYS Workbench 2019 R3. Tab.3 shows the thermal conditions in the simulation. Tab.4 shows the major temperature dependent material parameters of SUS316L given in ANSYS database.

\subsection{Simulation and experiment results}

Fig. 4 shows the substrate temperature measured by thermocouple 1 and the temperature obtained by the simulation. Three temperature peaks corresponding to the three beads were confirmed. The difference between the measured and simulated results was within $5 \%$ at the highest peak. A similar tendency was also seen in thermocouple 2. This result showed that the laser heat and its duration in the simulation were appropriately set.

Fig. 5 shows the simulated and measured therma displacements at line $A^{\prime}$. The displacement of the substrate in the $Z$ direction increases while the number of beads increases. Both measured and simulated displacements of three beads were approximately two times larger than those of one bead, respectively.

Fig. 6 shows the simulated and measured thermal displacements at line BB'. The measurement results for $y=18.0 \mathrm{~mm}$ to $y=25.5 \mathrm{~mm}$ were omitted because the measurement was interfered by the thermocouple cables. When the first bead was fabricated, the displacement was positively correlated with y position. However, when the

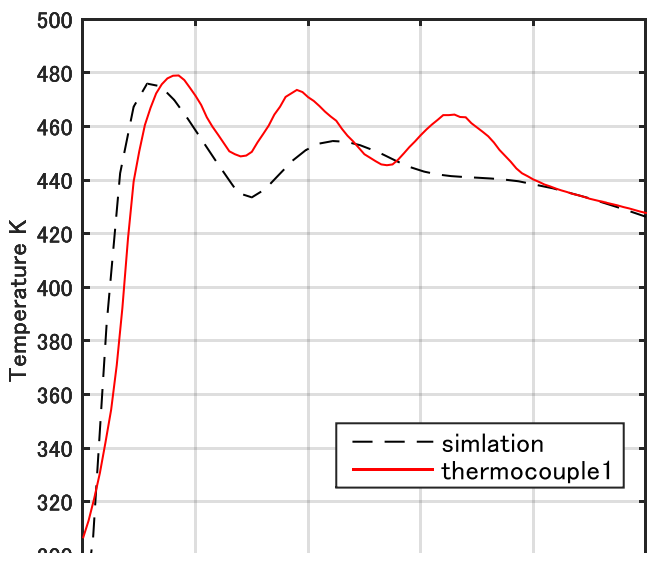

Fig.4: Comparison of measured and simulated temperature at Thermocouple1.

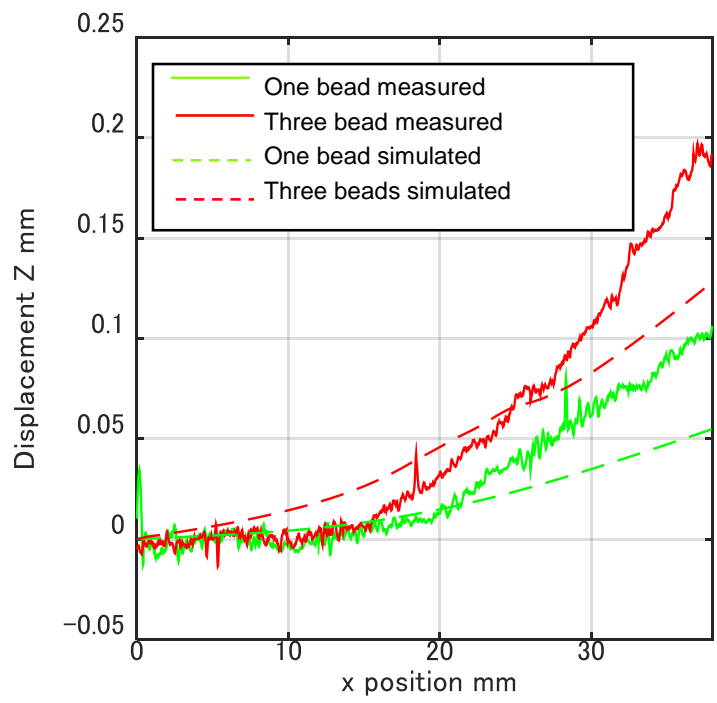

Fig. 5 : Substrate displacement of experiment and simulation on line $A A^{\prime}$ in plane fabrication.

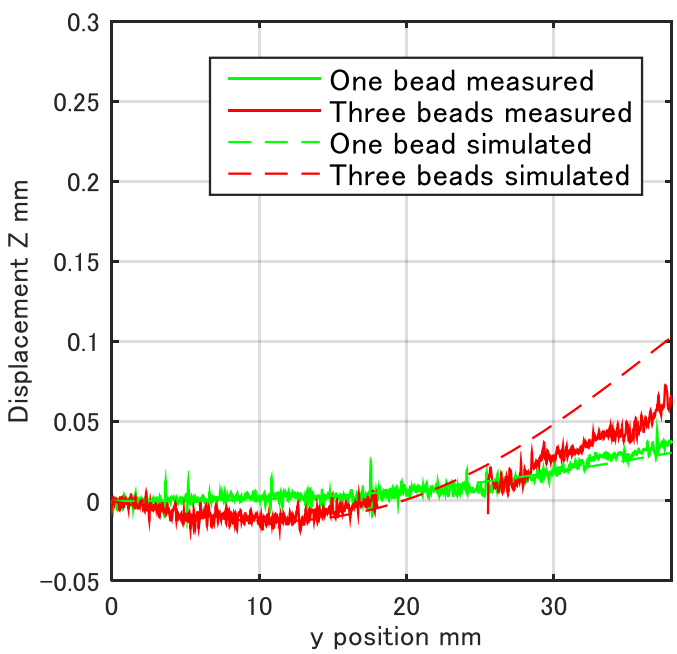

Fig. 6 : Substrate displacement of experiment and simulation on line BB' in plane fabrication. 
third bead was fabricated, the displacement had a minimum value both in the experiment and simulation.

The results of this section show that the thermal displacement increases as the number of beads increases in plane fabrication.

\section{EFFECTS OF OUT OF PLANE FABRICATION ON THERMAL DEFORMATION}

To investigate the effect of the out of plane fabrication on the thermal deformation, experiment and simulation were performed.

\subsection{Experimental and simulation method}

Fig. 7 shows the geometry of the substrate and fabricated workpiece. Five out of plane beads were fabricated on the upper surface of the substrate. The displacement of the upper surface of the substrate in the $\mathbf{Z}$ direction was obtained by experiment and simulation.

The substrate was fixed to a table of the machine with a M5 cap screw. The material of the substrate and table is JIS SUS316L.

Five beads were fabricated in the center of the substrate in order toward the positive $Z$ direction. The laser scanning by DED proceeded in the positive $Y$ direction. The profile of the substrate surface was measured by the manner similarly to that described in Section 2.2. The simulation was also conducted similarly to the manner described in Section 2.3

\subsection{Simulation and experimental results}

Fig. 8 (a) and (b) respectively show the simulated and measured thermal displacement on line $A A^{\prime}$. In the simulation, the deformation in the first bead accounted for most of total displacement of the fifth layer. The second and subsequent layers were hardly deformed. In the experiment, the deformation in the first bead was approximately $50 \%$ of the total displacement of the fifth layer.

Fig. 9 (a) and (b) respectively show the simulated and measured thermal displacement on line BB'. Similarly to the deformation along line $A A^{\prime}$, the deformation in the first and second beads was dominant.

From Fig. 9 (a), in the simulation, the deformation of the first bead was approximately $50 \%$ of the sum of the deformation up to the 5th layer. From the second layer onward, the deformation continued, although the deformation per one layer gradually decreased. From Fig. 9 (b), in the experiment, the deformation of the first layer accounted for

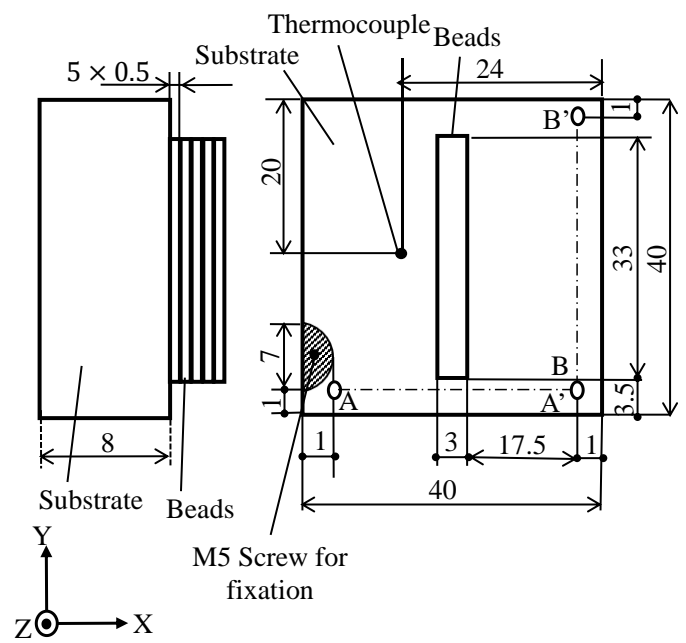

Fig. 7: Geometry of substrate and fabrication out of plane.

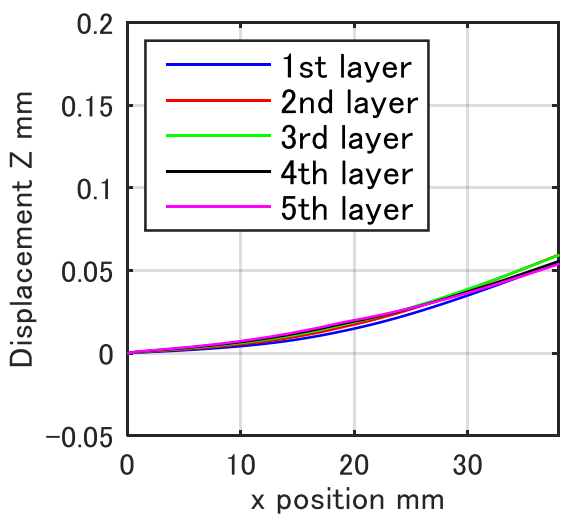

(a) Substrate displacement in simulation

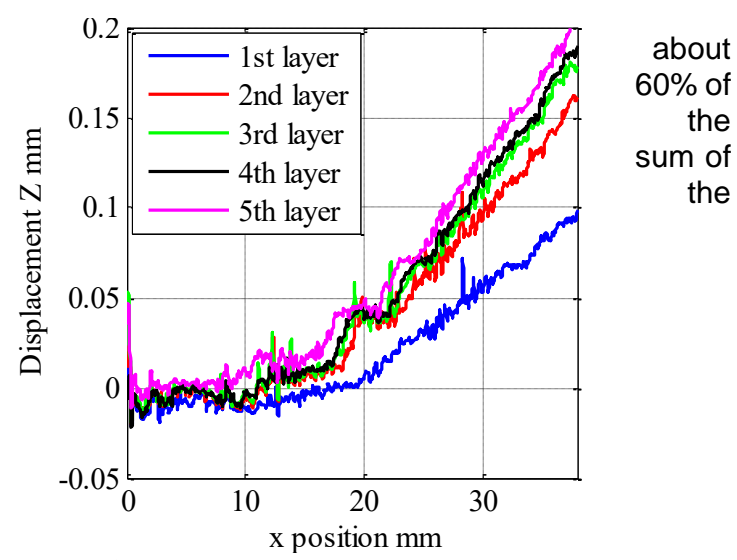

(b) Substrate displacement in experiment

Fig. 8 : Substrate displacement of each layer on line $A A^{\prime}$ out of plane fabrication.

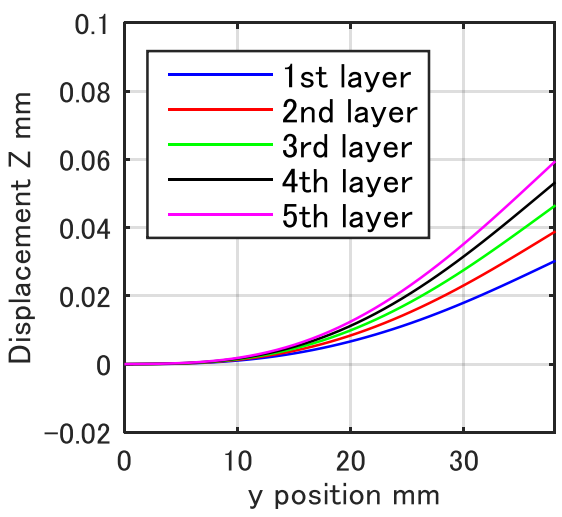

(a) Substrate displacement in simulation

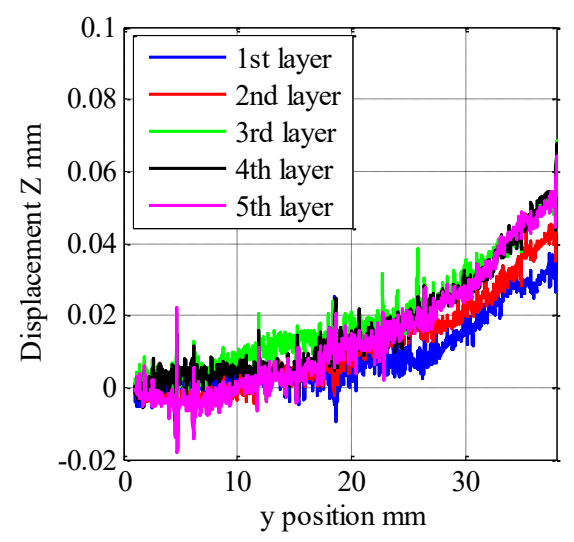

(b) Substrate displacement in experiment

Fig. 9 : Substrate displacement of each layer on line BB' out of plane fabrication. 
deformation up to the 5th layer. The deformation per each layer decreased similarly to the manner seen in Fig.9(a).

In Fig.8 and Fig.9, the variation of the experimental displacement could be caused by the variation of the laser absorption rate and geometry of the workpiece. The laser absorption rate of the workpiece is different between the substrate and bead. The geometry of the workpiece gradually changes from the flat to a rounded shape. These variations saturate by the 4th to 5 th layer at latest. Thus, the experimental displacement in the 1st to 3rd layer gradually changed.

The results of this section showed that in the out of plane fabricating, the deformation at the initial stage of fabrication had a significant effect on the total deformation.

\section{METHOD FOR REDUCING THERMAL DEFORMATION OUT OF PLANE DIRECTION BASED ON WELDING THEORY}

The discussion in Section 3 shows that the thermal deformation in a first bead is dominant when stacking multiple layers. Thus, in this section, a method for reducing the deformation at the first layer is proposed in consideration of the principles of thermal stress and thermal strain generation. Simulations are performed to verify the validity of the proposed method.

\subsection{Thermal Gradient method}

The principles of thermal stress and thermal strain generation are explained by Thermal Gradient Mechanism (TGM) in welding field [Shi 2007]. Fig. 10 shows the overview of thermal stress and thermal deformation in welding. A metal plate is welded along the center line (Fig. 10(a)). The heated part and the surrounding non-heated parts are approximated by a beam. Both ends of the three beams are connected to a rigid rod that can be freely displaced (Fig. 10(b)).

Fig. 11 shows the relationship between the stress and temperature of a center line considering elasto-plastic deformation. The symbols $T_{A}$ to $T_{D}$ represent the temperature at point $\mathrm{A}$ to $\mathrm{D}, \sigma_{y 0}$ represents the yield stress. In the heating state represented by arrow OA (see also Fig. 10 (c2)), compressive stress occurs in the heated part because of the thermal expansion of the heated part. Once the compressive stress reaches yield stress, plastic compressive deformation occurs in heated part because of the restriction of the non-heated part. This state is represented by arrows $A B, A C$ and $A D$ depending on the temperature rise.

In the cooling state represented by arrows BG, CF and $\mathrm{DE}$ (see also Fig. 10 (c3)), tensile stress occurs in the heated part because of the thermal expansion of the heated part. As represented by arrow EF, once the tensile stress reaches yield stress, plastic tensile deformation occurs in heated part because of the restriction of the non-heated part. The residual thermal deformation is generally caused by the plastic strain generated near the welded area. Thus, the compressive plastic strain after cooling should be reduced to reduce the thermal deformation.

The residual strain at the point $F$ is calculated when considering the cycle OADEF because the temperature near the laser spot is generally very high. The residual strain $\varepsilon_{F}$ at point $F$ is expressed by the following equation:

$\varepsilon_{F}=-\left(T_{\text {Max }}-T_{\mathrm{A}}\right) \alpha+\left(T_{\text {Max }}-T_{\mathrm{C}}\right) \alpha \quad\left(T_{C}<T_{\text {Max }}<T_{D}\right)$

where $T_{\mathrm{Max}}$ is the maximum temperature, $\alpha$ is the thermal expansion coefficient.

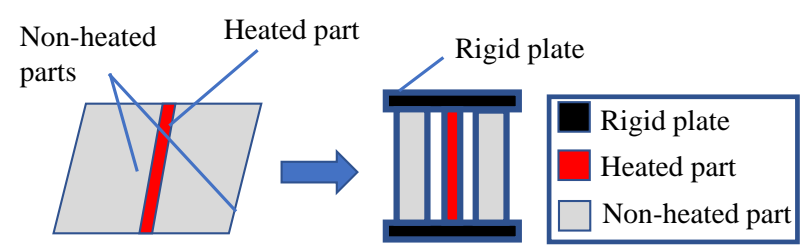

(a) Welded plate

(b) Modeled plate

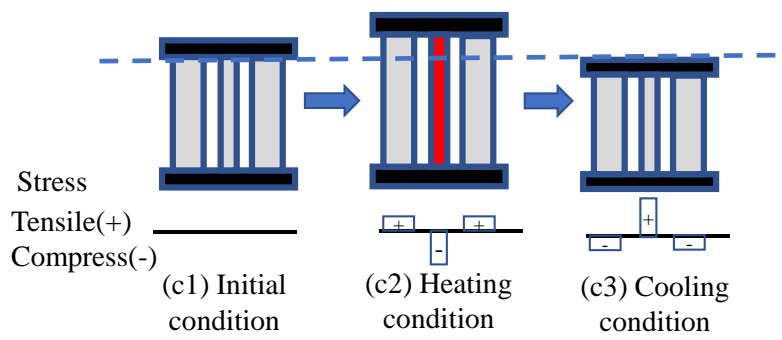

Fig. 10: Outline of thermal stress and thermal deformation in the welding.

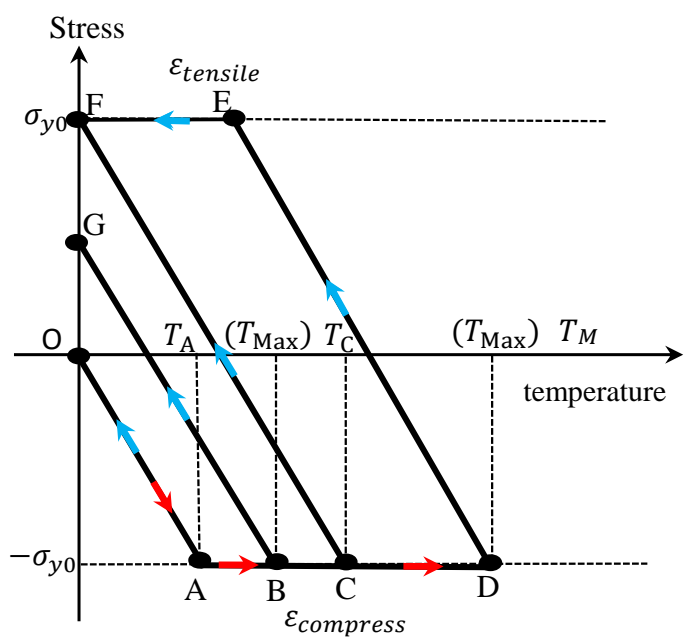

Fig. 11: Stress depending on temperature in elastoplastically deformed metal. Red arrows represent heating process. Blue arrows represent cooling process

The temperature $T_{A}$ was derived from the stress-strain relationship. The temperature $T_{C}$ was derived from the deformation from point $\mathrm{C}$ to point $\mathrm{F} . \mathrm{T}_{\mathrm{A}}$ and $T_{\mathrm{C}}$ are expressed by the following equations:

$A \sigma_{y 0}=\left(\alpha T_{A} L\right) k^{*} \frac{k}{k+k^{*}}$

$T_{A}=\frac{\sigma_{y 0}}{\beta_{\mathrm{H}} E \alpha}$

$\alpha T_{\mathrm{C}}=\frac{\sigma_{y 0}}{\beta_{\mathrm{C}} E}+\frac{\sigma_{y 0}}{\beta_{\mathrm{C}} E}$

$T_{\mathrm{C}}=\frac{2 \sigma_{y 0}}{\beta_{\mathrm{C}} E \alpha}$

where $E$ is the elastic modulas, $k$ and $k^{*}$ are the rigidity of heated part and non-heated part respectively, $\beta_{\mathrm{H}}$ and $\beta_{\mathrm{C}}$ are the degree of restraint during heating and cooling, respectively, which are obtained by the following equations:

$\beta=\frac{k^{*}}{k+k^{*}}$

$k=E A / L$

$k^{*}=E^{*} A^{*} / L$

where $E$ and $A$ are the elastic modulus and cross section of the heated part, respectively and $E^{*}$ and $A^{*}$ are the elastic 
modulus and the cross section of the non-heated part, respectively.

The residual strain at point $F$ is expressed by substituting Eq.(3) and (4) into Eq. (2):

$\varepsilon_{F}=\frac{\sigma_{y 0}}{E}\left(\frac{1}{\beta_{\mathrm{H}}}-\frac{2}{\beta_{\mathrm{C}}}\right)$.

$$
\left(T_{C}<T_{\operatorname{Max}}<T_{D}\right)
$$

For simplicity, $\sigma_{y 0}, E$, and $\alpha$ are assumed to be constant regardless of temperature.

\subsection{Method to reduce thermal deformation}

From Eq. (2), the residual strain was obtained by the sum of the strain caused in AD and EF in Fig. 11. The strain caused in $A D$ is compressive plastic strain and the strain caused in EF is tensile plastic strain. Thus, the residual strain can be suppressed by cancelling the strain in AD and $E F$. At a predetermined maximum temperature, the strain in $A D$ and EF is influenced by Beta as expressed by Eq.(8). When $\beta_{\mathrm{H}}$ and $\beta_{\mathrm{C}}$ are equal, the strain in $\mathrm{AD}$ is larger than that in EF. In our study, we tried to increase $\beta_{\mathrm{C}}$ to increase the strain in EF.

we try to increase $\beta_{\mathrm{C}}$ by keeping the stiffness $k^{*}$ of the nonheated part. In the cooling state, $E^{*}$ is decreased due to the residual temperature rise. Thus, the decrease of $E^{*}$ can be at minimum by keeping the residual temperature rise of the non-heated part as small as possible. An additional cooling of the non-heated part can be an effective solution. In this study, the effect of the interval for cooling between depositions on the thermal deformation is investigated in the following part. A long interval leads to the smaller temperature rise of the non-heated part.

\subsection{Simulation method}

The effect of the proposed method was verified by simulation which was similar to that described in Section 2.3. The thermal deformation of the workpiece in the $Z$ direction was obtained with different interval for cooling between depositions. The interval was set to $2.4 \mathrm{~s}$ in the conventional fabrication and $242.4 \mathrm{~s}$ in the proposed method.

\subsection{Result of the simulation and discussion}

Figure 12 shows the thermal displacement in the $Z$ direction on line BB'. The curvature of the displacement was larger for the conventional method. The difference between the maximum and minimum displacements was $0.115 \mathrm{~mm}$ with the conventional method and was $0.0762 \mathrm{~mm}$ with the proposed method. Thus, the thermal deformation was reduced by $34 \%$ by the proposed method.

Figure 13 shows the thermal displacement on line AA'. The displacement is positively correlated with $x$ position and was approximately $0.13 \mathrm{~mm}$ at $x=38 \mathrm{~mm}$. The conventiona method and proposed method showed similar results in

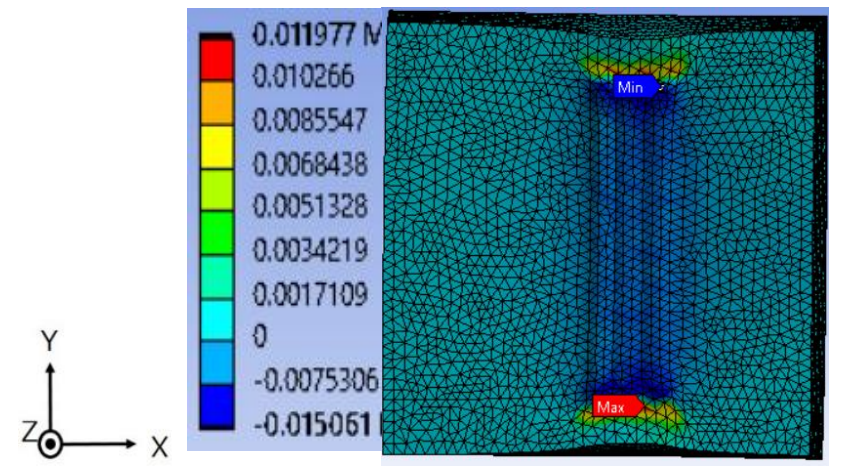

(a) Plastic strain in conventional method overview although the $0.0061 \mathrm{~mm}-0.016 \mathrm{~mm}$ difference was observed in $x$ position of $10-30 \mathrm{~mm}$.

The reason why there is no difference between the conventional and proposed methods can be explained by the TGM. When the laser is scanned along the $Y$ direction, three beams shown in Fig. 10 are parallel to the $X$ direction.

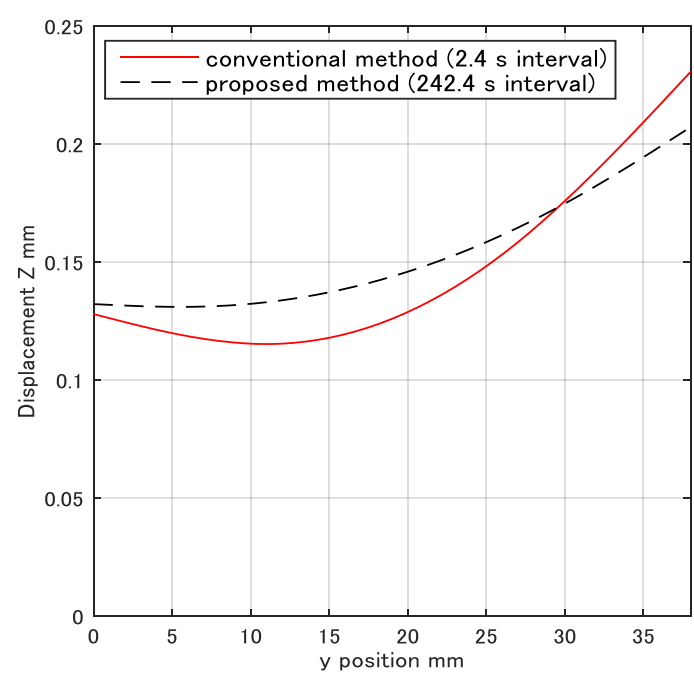

Fig. 12 : Thermal displacement of the two conditions on the $B B^{\prime}$ line

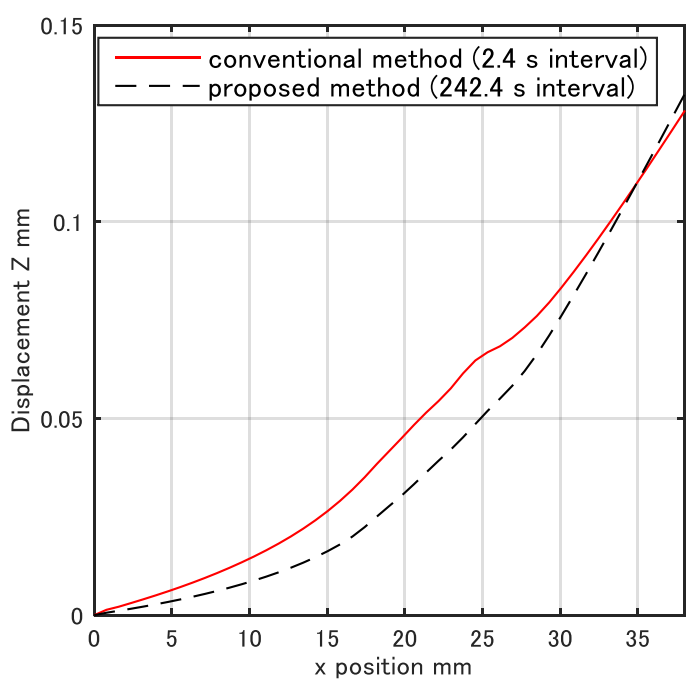

Fig. 13 : Thermal displacement of the two conditions on the $A A^{\prime}$ line

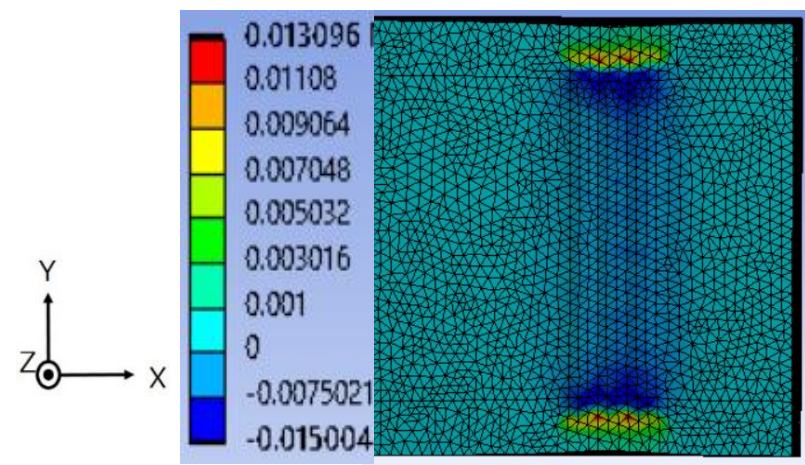

(b) Plastic strain in proposed method

Fig. 14 : Color map of the plastic strain in different interval for cooling. 
Because the laser passes laterally on the three beams, the temperature distribution of the three beams during cooling is almost the same. This causes an equivalent shrinkage of the beams which results in the small thermal deformation difference in the $X$ direction.

Figures 14 (a) and (b) show the color map of the plastic strain of the substrate surface in the $Y$ direction. The blue color represents the compressive plastic strain, and the red color represents the tensile plastic strain. The maximum compressive plastic strain is almost equal in both methods. However, the blue area showing the compressive plastic strain is smaller in the proposed method than in the conventional method. Therefore, the proposed method can reduce the plastic strain and the thermal displacement.

\section{CONCLUSION}

In this paper, the workpiece thermal deformation in metal additive manufacturing was investigated. Experiments and simulations were conducted to investigate the thermal deformation in the out of plane direction during the out of plane and in plane fabrication, which are the typical manufacturing pattern in the additive manufacturing technology. Then, based on the above investigation, a method to reduce thermal deformation was proposed considering TGM. The conclusions obtained in this paper are as follows.

1. In the in plane fabricating, the deformation for each bead was summed up. In the out of plane fabrication, the deformation at the first bead was dominant.

2. A method to suppress the workpiece deformation in the out of plane direction was proposed based on TGM theory. The temperature of the area surrounding the fabrication spot should be decreased to reduce the thermal deformation of the workpiece.

3. The simulation results showed that the proposed method was effective in suppressing deformation in the out of plane direction along the lateral direction. The thermal deformation was reduced by $34 \%$ by the proposed method.

\section{REFERENCES}

[Klocke 2014] Klocke F., Klink A., Veselovac D., Keith D.A., Soo S.L., Schmidt M., Schilp J., Levy G., Kruth J.P., Turbomachinery component manufacture by application of electrochemical, electro-physical and photonic processes, CIRP Annals, 2014, Vol.63, pp.703-726, ISSN 0007-8506.
[Bartolo 2012] Bartolo P, Kruth J.P., Silva J., Levy G., Malshe A., Rajurkar K., Mitsuishi M., Ciurana J., Leu M., Biomedical production of implants by additive electrochemical and physical processes, CIRP Annals, 2012, Vol.61, pp.635-655, ISSN 0007-8506.

[Thompson 2016] Thompson M.K., Moroni G., Vaneker T., Fadel G., Campbell R.I., Gibson I., Bernard A., Schulz J., Graf P., Ahuja B., Martina F., Design for Additive Manufacturing: Trends, opportunities, considerations, and constraints, CIRP Annals, 2016, Vol.65, pp.737-760, ISSN 0007-8506.

[Li 2018] Li Y., Zhou K., Tan P., Tor S.B., Chua C.K., Leong K.F., Modeling temperature and residual stress fields in selective laser melting, International Journal of Mechanical Sciences, 2018, Vol.136, pp. 24-35, ISSN 0020-7403.

[Zhang 2018] Zhang H., Xu W., Xu Y., Lu Z.\& Li D., The thermal-mechanical behavior of WTaMoNb high-entropy alloy via selective laser melting (SLM): experiment and simulation, International Journal of Advanced Manufacturing Technology, 2018, Vol.96, pp. 461-474.

[Cheng 2016] Cheng B., Shrestha S., Chou K., Stress and deformation evaluations of scanning strategy effect in selective laser melting, Additive Manufacturing, 2016, Vol.12, Part B, pp.240-251, ISSN 2214-8604.

[Simson 2017] Simson T., Emmel A., Dwars A., Böhm J., Residual stress measurements on AISI $316 \mathrm{~L}$ samples manufactured by selective laser melting, Additive Manufacturing, 2017, Vol.17, pp.183-189, ISSN 22148604.

[Dong 2014] Dong P., Song S., Zhang J., Analysis of residual stress relief mechanisms in post-weld heat treatment, International Journal of Pressure Vessels and Piping, 2014, Vol.122, pp.6-14, ISSN 0308-0161.

[Verhaeghe 1999] Verhaeghe G., Predictive formulae for weld distortion: a critical review, Woodhead Publishing, 1999, ISBN: 9781855734449

[Kruth 2003] Kruth, J.P., Wang, X., Laoui, T. and Froyen, L., Lasers and materials in selective laser sintering, Assembly Automation, 2003, Vol. 23, pp. 357-371.

[Shi 2007] Shi Y., Shen H., Yao Z., Hu J., Temperature gradient mechanism in laser forming of thin plates, Optics \& Laser Technology, 2007, Vol.39, pp.858-863, ISSN 00303992. 